\title{
El gobierno electrónico en América Latina desde una perspectiva crítica'
}

\author{
Ángel Páez \\ (Universidad del Zulia-Venezuela)
}

Recibido: 6/11/2011

Aprobado: 10/12/2011

\begin{abstract}
Resumen: El artículo se basa en el estudio de la situación del gobierno electrónico (GE) en Venezuela en los últimos diez años (Páez, Iribarren y Neüman 2003; Páez y Castañeda 2007; Montilla y Páez 2007 y 2010; Páez 2010 y 2011), para proponer lineamientos teóricos que podrían contribuir a generar una discusión orientada al desarrollo de teorías latinoamericanistas sobre el fenómeno del gobierno electrónico, así como a reorientar la política pública en esa materia.
\end{abstract}

Palabras clave: gobierno electrónico / TIC / participación ciudadana

\section{E-government in Latin America from a critical perspective}

Summary: The article is based on the study of e-government situation in Venezuela in the last ten years (Páez, Iribarren and Neuman 2003; Páez Castañeda 2007; Montilla and Páez 2007 and 2010, Páez 2010 and 2011), to propose theoretical guidelines that could help generate a discussion focused on the development of Latin American theories about the phenomenon of electronic government and to redirect public policy in this area.

Keywords: e-government / ICT / citizenship

1 Producción intelectual del proyecto de investigación “El gobierno electrónico en los espacios autónomos estratégicos (EAE) para la participación ciudadana sustentada en la apropiación social", cuyo investigador responsable es Ángel Páez, incluido en el Programa de Investigación "Experiencias locales de apropiación tecnológica Parte III", adscrito al Centro de Investigación de la Comunicación y la Información (CICI) de la Universidad del Zulia (LUZ) y financiado por el Consejo de Desarrollo Científico y Humanístico de LUZ bajo el № VAC-CONDES- CH-0529-10. 


\section{A manera de introducción}

El gobierno electrónico puede definirse en términos tan simples como fines públicos por medios digitales. Subyacen dos expectativas sobre este: la posibilidad de ofrecer servicios vía electrónica y la de desarrollar democracia electrónica. En el primer sentido, se espera que el gobierno pueda proveer servicios a los ciudadanos por medio de mecanismos electrónicos que permitan mejorar la calidad de la entrega, requiriéndose transparencia y acceso a la información. En un segundo sentido, en el cual se tienen mayores expectativas, es cuando el gobierno electrónico constituye un espacio donde los ciudadanos se reúnen en igualdad de condiciones para debatir sobre temas que sean significativos para la comunidad y para tomar decisiones que dan forma a la sociedad (Reilly y Echeverría 2003).

La propuesta del Estado venezolano aparece siguiendo esta concepción de gobierno electrónico en la cual el propósito de su aplicación para la participación ciudadana es prioritario sobre otros enfoques de gobierno electrónico, como los de Criado, Ramilo y Salvador (2002), Backus (2001), OkotUMA (2001), Holmes (2001), quienes parten de una perspectiva eurocéntrica y neoliberal que persigue desarrollar el gobierno electrónico en función de los intereses del mercado.

Se podría afirmar que el marco legal creado para la aplicación del gobierno electrónico en Venezuela hace énfasis en los aspectos que contribuyen a que el ciudadano pueda desarrollar un rol protagónico. Ejemplo de ello lo encontramos en la Constitución de la República Bolivariana de Venezuela, que promueve la participación ciudadana como el principal medio por el cual el pueblo ejerce su soberanía, así como la Ley del Estatuto de la Función Pública, la Ley Orgánica de la Contraloría General de la República y del Sistema Nacional de Control Fiscal (2001), la Agenda de Gobierno Electrónico de Venezuela (2001), el Nuevo Mapa Estratégico de la Presidencia de la República Bolivariana de Venezuela (2004), el Decreto 825 (2000), el Plan-TIC 20072013, el Decreto 3.390 (2004), la Ley Orgánica de Ciencia, Tecnología e Innovación (2001), la Ley de Telecomunicaciones (2000), el Proyecto de Ley de Infogobierno, la Ley Orgánica del Poder Público Municipal (2006), las normas para fomentar la participación ciudadana (2007), la ley contra la corrupción (2003), la Ley Orgánica de Planificación (2001) y la Ley de los Consejos Comunales (2006), entre otros.

De acuerdo con la Constitución venezolana, la participación ciudadana es el eje fundamental de la Democracia, ya que representa un mecanismo o instrumento indispensable para acceder al poder. Es por medio de la participación ciudadana que se puede construir ciudadanía, pero entendida en términos de Salazar (2005: 58), quien apunta a un sujeto "[...] que 
se asume con derechos y capacidad para re-situarse en el espacio público, revelándose como sujeto desciudadanizado y exigiendo una ciudadanía con derechos, donde los derechos lo coloquen en posibilidad de ejercitar sus responsabilidades".

Sin embargo, se puede afirmar que el gobierno electrónico en Venezuela se encuentra apenas en la fase informacional, atendiendo a la clasificación de Gartner (2000) según varios estudios realizados en el país en los últimos años (Rincón y Romero 2003, Gonzalo 2005 y 2006, Ochoa 2006, Montilla y Páez 2006, Páez y Castañeda 2007, entre otros).

Esto significa que aunque una primera etapa ha sido desarrollada con la ventaja de contar además con todo un marco legal para su aplicación, el proceso de gobierno electrónico en Venezuela tiene por delante un largo camino que recorrer antes de poder alcanzar plenamente su propósito de impulsar la participación ciudadana. Para lograrlo, necesitamos superar la visión del Estado basada en la eficiencia técnica y avanzar hacia un modelo menos consumista de tecnología que tienda a resolver problemas básicos de la sociedad (Andrade 2009).

Cendrós, Durante y Fermín (2004) advierten que en las alcaldías de Venezuela no hay voluntad política, ni una política informática orientada al pensamiento estratégico en los desarrollos de gobierno electrónico.

Si medimos al gobierno electrónico en Venezuela de acuerdo con la participación electrónica (plan de acción de gobierno electrónico, desarrollo de estrategias y políticas de participación electrónica y el estado de las TIC en el país), encontramos que en el 2005 Venezuela ocupaba una posición privilegiada entre los primeros 20 países de Latinoamérica, ubicándose en el puesto siete (7) (tabla 1).

Tabla 1

Informe sobre el gobierno electrónico en Sudamérica y América Central

\begin{tabular}{lcccc}
\hline & Índice & \multicolumn{2}{c}{ Clasificación } & Modificación \\
& $\mathbf{2 0 0 5}$ & $\mathbf{2 0 0 5}$ & $\mathbf{2 0 0 4}$ & \\
\hline Chile & 0,6963 & 22 & 22 & 0 \\
México & 0,6061 & 31 & 30 & -1 \\
Brasil & 0,5981 & 33 & 35 & 2 \\
Argentina & 0,5971 & 34 & 32 & -2 \\
Uruguay & 0,5387 & 49 & 40 & -9 \\
Colombia & 0,5221 & 54 & 44 & -10 \\
Venezuela & 0,5161 & 55 & 56 & 1 \\
Perú & 0,5089 & 56 & 53 & -3 \\
Panamá & 0,4822 & 64 & 54 & -10 \\
\hline
\end{tabular}

(continúa) 
(contínuación)

\begin{tabular}{lcccc}
\hline & Índice & \multicolumn{2}{c}{ Clasificación } & Modificación \\
& $\mathbf{2 0 0 5}$ & $\mathbf{2 0 0 5}$ & $\mathbf{2 0 0 4}$ & \\
\hline Costa Rica & 0,4612 & 70 & 73 & 3 \\
El Salvador & 0,4225 & 78 & 79 & 1 \\
Bolivia & 0,4017 & 85 & 88 & 3 \\
Guyana & 0,3985 & 89 & 71 & -18 \\
Ecuador & 0,3966 & 92 & 82 & -10 \\
Belize & 0,3815 & 97 & 76 & -21 \\
Guatemala & 0,3777 & 100 & 111 & 11 \\
Paraguay & 0,3620 & 107 & 109 & 2 \\
Surinam & 0,3449 & 110 & 105 & -5 \\
Nicaragua & 0,3383 & 113 & 121 & 8 \\
Honduras & 0,3348 & 115 & 113 & 2 \\
Promedio & 0,4643 & & & \\
\hline
\end{tabular}

Fuente: United Nations (20005). Global E-government Readliness Report.

La posición ventajosa de Venezuela a principios de la década en materia de participación electrónica obedece a la orientación social de la agenda de gobierno electrónico, en la que se observa, de acuerdo con Reilly (2004), la intención de transformar radicalmente el Estado y se reconocen, al menos indirectamente, el enfrentamiento y los conflictos que puedan presentarse entre la gestión pública tradicional enmarcada en la democracia representativa de corte neoliberal y la construcción de una democracia directa, participativa y sustantiva. A juicio de Reilly
(2004) "la definición de Venezuela es la que pone el mayor énfasis en los aspectos socialmente relevantes del e-gobierno".

Las estadísticas en el 2010 muestran un balance diferente. De acuerdo con el índice de la ONU, en cuanto a participación electrónica Venezuela se muestra lejos del líder regional, que es Colombia (tabla 2). Si bien no se puede hablar de retroceso, hay un evidente estancamiento que tiene un gran significado para un país que hoy debería liderar los avances de gobierno electrónico en América Latina. 
Tabla 2

Índice de participación electrónica

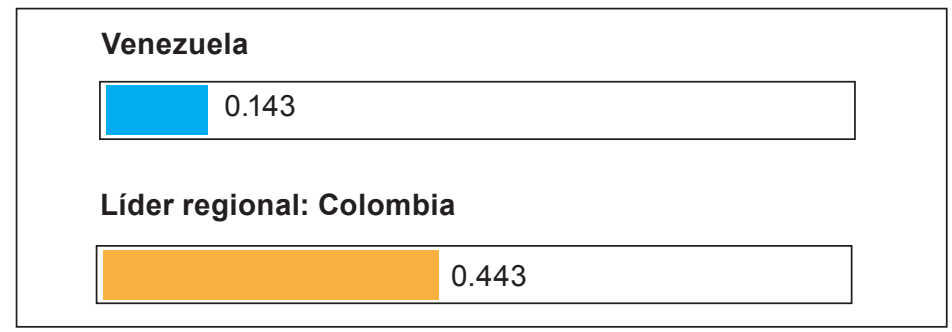

Fuente: United Nations (20005). Global E-government Readliness Report

Coincidimos con Albornoz (2007), quien plantea que el estancamiento se debe fundamentalmente a que durante la primera década del siglo XXI el despliegue de la Agenda Nacional de Gobierno Electrónico no obedeció a su carácter social sino a una lógica instrumental -desde un modelo de negocios- que ha convertido al Estado en un consumista de tecnología, prestando demasiada atención a las soluciones técnicas y mucho menos a la complejidad de los desafíos sociales que implica profundizar los niveles de apropiación social de las tecnologías de la información y la comunicación (TIC). ${ }^{2}$

Por los estudios de Albornoz (2007), Páez y Castañeda (2007), Páez y Montilla (2010), Gotopo, Leal y
Oliveros (2010), De Barry y Palmar (2010), entre otros, no es difícil concluir que en la actualidad el gobierno electrónico venezolano debe hacer mayores esfuerzos para propiciar la democracia. Para lograrlo, debe entenderse que cuando los venezolanos participan usando la tecnología, lo hacen a través de procesos de apropiación. La tecnología penetra el tejido social y ello provoca actos de apropiación que ocurren de manera intencional y con carácter social, cultural, económico y político. "Apropiarse es un acto dentro de la esfera de la subjetividad del que se apropia. Lo que se apropia le será desde ese momento propio... más en significados, usos y propósitos que en el aspecto de la propiedad" (Neüman 2008: 65).

2 Al respecto, recomendamos la lectura del artículo "Twitter y Blackberry son una moda, la atención a la gente no puede estar construida sobre una moda" de Luigino Bracci Roa: $<$ http://www.aporrea.org/actualidad/a100336.html $>$. 
Se presenta entonces un escenario en el que se debe reorientar la política pública sobre gobierno electrónico, y surge la pregunta: ¿cuáles deben ser las categorías a considerar en esa nueva política pública? En este artículo adelantamos las respuestas a esas preguntas por medio del análisis de las teorías que han predominado en las agendas públicas sobre gobierno electrónico en América Latina.

\section{Propuestas para y desde América Latina}

En cada Estado-nación deben generarse teorías propias que permitan diseñar y ejecutar políticas públicas enmarcadas en los enfoques políticos y epistemológicos que correspondan con la dinámica del mundo social que da sentido y pertinencia a los gobiernos.

En una búsqueda -inacabada- de una teoría propia y venezolana sobre el gobierno electrónico como política pública prioritaria del Estado venezolano, encontramos a varios latinoamericanos como Andrade (2007, 2009), Kaufman y Piana (2007), Sandoval (2009), Neüman (2008, 2009), Albornoz (2007), García Santiago (2009), Cendrós, Durante y Fermín (2004), Cardon (2005), Araya (2004 y 2005), Reilly (2004), Petrizzo (2005), Zavarce (2005), Gonzalo (2006), Carballo, Cattafi, Sanoja y Zambrano (2006), quienes ya han adelantado el camino de la urgente reflexión teórica y política que amerita el problema del gobier- no electrónico, dada la relevancia estratégica que hoy representan las TIC como palanca para la transformación social y el desarrollo.

En principio debemos establecer que la esencia de la discusión sobre el papel de las TIC en las relaciones Estado-ciudadanía (gobierno electrónico) no debe ser tecnológica ni orientada exclusivamente a la eficiencia y eficacia en la prestación de servicios (ya que eso correspondería al enfoque instrumental), sino más bien enmarcada en los procesos de reconstrucción de la democracia. Consideramos que internet, además de ser un espacio para el tránsito mediatizado de los intereses del mercado, puede ser al mismo tiempo y paradójicamente una forma de mediación que hoy están utilizando los ciudadanos para formar "sus propias constelaciones políticas e ideológicas, evitando las estructuras políticas establecidas y creando un ámbito político adaptable" (Morales 2004: 76).

Araya (2005: 56) expresa de igual forma que "[...] internet puede ser vista como una posibilidad de empoderamiento para el ciudadano, o por otro lado, como fábrica de hegemonía para las empresas y los gobiernos". De tal manera que el gobierno electrónico se desarrolla justo en el filo de la navaja entre el modelo de administración pública instrumental y unidireccional (correspondiente a la democracia representativa) y una nueva forma de gestión pública centrada en el ciuda- 
dano (democracia participativa y protagónica).

En las teorías foráneas observamos la separación entre gobierno electrónico y democracia electrónica, enmarcados ambos en la gobernanza electrónica. Desde ese enfoque, el gobierno electrónico es una herramienta para satisfacer a los clientes-ciudadanos, ahorrar en recursos e integrarse más armoniosamente con el mercado a través del comercio electrónico (e-commerce). Coincidimos con Araya (2005) en dos argumentos: 1) en la discusión, no debe separarse el gobierno electrónico de la democracia electrónica, y 2) el énfasis no debe estar en lo electrónico, sino en una discusión esencialmente política sobre los fundamentos de la democracia. "El desafío es lograr establecer un vínculo entre gobierno electrónico y democracia electrónica a fin de ir más allá de los modelos unidireccionales de servicio y políticas públicas" (Araya 2005).

Y si el énfasis de la discusión debe estar en los fundamentos de la democracia, conviene entonces considerar los niveles de intensidad de participación ciudadana que proponen Coleman y Gotze (2001, citados por Araya 2005: 66):

[...] democracia electrónica de opinión pública, donde se consulta a la población a través de testeos de opinión, pero sin ningún compromiso resolutivo. Se trata de utilizar internet para escuchar a la gente. Un segundo nivel es la democracia directa o plebiscitaria, donde se procede a consultar por diversas materias de interés a través de internet, a diferencia de los levantamientos de opinión, con un carácter resolutivo. En tercer lugar los autores señalan un modelo de democracia electrónica basada en comunidades locales, donde las organizaciones de base territorial o funcional adquieren nuevas herramientas para interactuar con el municipio, con el gobierno central o con otras organizaciones. El cuarto modelo es el de compromiso cívico en la deliberación politica (online public engagement in policy deliberation). Éste es el que los autores presentan como el más complejo y democrático. El énfasis está en los elementos deliberativos de la democracia (las cursivas son nuestras).

En el caso venezolano, la evidencia empírica de Albornoz (2007), Páez y Castañeda (2007), Páez y Montilla (2010), Raenyra y Díaz (2010), Gotopo, Leal y Oliveros (2010), De Barry y Palmar (2010), Gonzalo (2005), Zambrano (2008) y Montilla y Páez (2006), demuestra que es mayor la fuerza de la ciudadanía que la del mismo gobierno al momento de avanzar en el desarrollo del gobierno electrónico. Esto podría indicar que, aunque parezca contradictorio, en la sociedad venezolana la transformación podría venir desde abajo, es decir, en los modelos de democracia electrónica basada en comunidades locales y el de compromiso cívico en la deliberación política. En el pulso político que hoy libran el gobierno y la ciudadanía, parece estar ganando la multitud.

Surge, entonces, la pregunta: ¿desde dónde enfocar el gobierno electró- 
nico? Reilly (2004) señala cuatro enfoques principales de gobierno electrónico: ${ }^{3}$ mejoras en la gestión de la administración pública, provisión de servicios, transparencia y facilitación de participación ciudadana. Los cuatro enfoques son unidireccionales e implican que la transformación comienza por el gobierno, pero como venimos sugiriendo en este trabajo, habría que pensar en un gobierno electrónico por el pueblo (democracia participativa y directa) y para el pueblo (democracia social o sustantiva).

Una aproximación al e-gobierno al estilo "Ford", de "proveer acceso a los efectos de crear un mercado para los servicios gubernamentales", no es un medio adecuado para facilitar la creación y sostenimiento de una ciudadanía creativa, curiosa, innovadora e interesada. Más bien, si ese es el objetivo, entonces la programación en Internet debe poner atención en suministrar contenido en línea, además de ambientes en línea que faciliten el acceso al "qué" y al "cómo" del gobierno (Reilly 2004: 101).

Además, señala Petrizzo (2005: 90), en el modelo agencial ${ }^{4}$ existe un riesgo latente de convertirlo en comercio electrónico y hacer de los ciudadanos consumidores de servicios gubernamentales". Al respecto, Zavarce (2005: 3) indica que:
[...] el "Gobierno Electrónico" no es un problema técnico, o de incorporación de mejores prácticas gerenciales a la Administración Pública, si no que, por el contrario, es un asunto epistémico. Es decir, se hace necesario en esta etapa del proceso de transformación de la Administración Pública "Gerenciar el Cambio", para entender el concepto. Transmitir con contundencia que la razón de ser de la idea que subyace a la propuesta de Gobierno Electrónico es la de pensar en "Transformación", y no como es planteado en el ámbito empresarial, como si se tratase de "una nueva práctica gerencial".

Lo que sí debe hacer el Estado es diseñar y ejecutar políticas públicas socialmente relevantes que propicien procesos emancipatorios en los que la ciudadanía pueda rebasar la barrera del acceso a las TIC, para apropiarse socialmente de ellas.

El diseño de políticas públicas debe partir de un enfoque social y amplio que permita advertir las paradojas que encierra el problema del uso de las TIC. Paramio (2000) advierte el peligro de asumir posturas extremas, al momento de examinar con algún detalle cuál podría ser la importancia real de los medios (entre ellos internet) en la vida democrática; por su parte, los apocalípticos parten de una concepción determinista del papel de la

3 De acuerdo con el análisis que efectuó a la agenda de GE en ocho países de América Latina.

4 En este modelo el gobierno se comporta como una agencia bancaria centrada en las necesidades de los clientes-ciudadanos. 
tecnología en el cambio social: una vez que existe es inevitable que el debate y la información sobre cuestiones políticas tiendan a enfocarse desde la lógica del entretenimiento, o que las personas dediquen cada vez más tiempo a usarla, abandonando la participación en asociaciones voluntarias y en actividades cívicas. Los integrados son ajenos a este determinismo: una vez que existe internet sería inevitable que los ciudadanos recurrieran cada vez más a la red para participar políticamente en la toma de decisiones sobre cuestiones públicas o para relacionarse con la administración e informarse sobre las actuaciones de los gobiernos y controlar a estos.

Como lo señalan Andrade y Yedra (2007), habría que empezar a enfocar el gobierno electrónico desde la teoría de los sistemas. Desde esta perspectiva el gobierno electrónico funciona como un sistema transparente que permite regular la acción pública a través de la ciudadanía.

Hace falta, por lo tanto, una visión del Estado distinta a aquella basada en la eficiencia técnica, que conduce al indefectible camino de construir un gobierno electrónico basado en lo tecnológico, para proponer un modelo menos consumista de tecnología que tienda a resolver problemas básicos de la sociedad, sustentado en el desarrollo de sistemas de información más eficientes en términos de la participación social y la calidad de los datos (Andrade y Yedra 2007: 84).
Habría también que superar la dicotomía que plantea la mayoría de los teóricos sobre la relación entre el gobierno y otros actores, ya que ello corresponde a la lógica instrumental que rechazamos en este trabajo. Tal es el caso de las relaciones G2G, G2C, G2E y G2B (tabla 3). En nuestra propuesta, el gobierno electrónico no está determinado por el Estado, sino por la ciudadanía, lo cual se traduce en un nuevo modelo de Estado constituido por el tercer sector.

Tabla 3

Relación gobierno-actores

\begin{tabular}{|l|}
\hline G2G $=$ De gobierno a gobierno \\
\hline G2C $=$ De gobierno a ciudadano \\
\hline G2E $=$ De gobierno a trabajadores \\
\hline G2B $=$ De gobierno a mercado
\end{tabular}

Elaboración propia.

\section{Consideraciones finales}

No pretendemos que lo propuesto tenga un carácter definitivo y acabado. Vemos este trabajo como una agenda a seguir en la misión de resituar la discusión teórica sobre la relación entre tecnología, sociedad y Estado. No convienen las perspectivas que aíslen el pensamiento político de los desarrollos teóricos en materia de tecnología, sobre todo si se trata de tecnologías (TIC) que atraviesan el tejido social mediante formas que incluso aún no han sido bien estudiadas. De manera que 
al equipo de investigación que respalda este trabajo le quedan pendientes diferentes líneas de acción: a) publicar el trabajo para su discusión con pares de diversas disciplinas que aporten en la maduración de la propuesta; b) propiciar espacios de divulgación en los que puedan acercarse actores sociales, investigadores, servidores públicos y líderes políticos que influyan en el diseño de políticas públicas en materia de gobierno electrónico; c) desarrollar una línea de investigación que permita sistematizar datos cualitativos y cuantitativos desde la perspectiva de la emergencia, el carácter móvil y los procesos de reapropiación social.

Queda también pendiente una revisión del término "gobierno electrónico", con el propósito de desarrollar una categoría que sea más cónsona con las condiciones propuestas en este trabajo. Consideramos que lo electrónico debe ampliarse en función de la evolución del hipertexto y de las redes sociales. En cuanto al término gobierno, habría que proponer una categoría que esté más orientada al poder y menos al control de un grupo sobre otros.

Nos embarga una pregunta inquietante: ¿qué tan viable es la propuesta en Venezuela? Se nos presentan varios escenarios inciertos: a) un tercer sector poco desarrollado; b) una clase política aún influenciada por el paradigma instrumental de la tecnología; c) estructuras estatales aún muy burocráticas y alejadas de la visión compleja; d) un mercado que ha penetrado la po- lítica pública en materia de TIC para inundarla con su modelo consumista.

Sería, entonces, muy atrevido apostar ciegamente por la viabilidad de la propuesta. Pero, por el otro lado, consideramos que la vía más ética para los investigadores latinoamericanos es la de la esperanza y la del riesgo de proponer lo que debería y podría pasar si se lograra una correlación de fuerzas adecuada entre la academia, la sociedad civil y el Estado.

Este trabajo deja constancia de la existencia de investigadores críticos altamente preocupados por el desarrollo social, científico y tecnológico en consonancia con una nueva visión de la sociedad que no se fundamente en los intereses de los grupos de poder (tanto en el mercado como en el Estado), sino en la participación como encuentro que ha propuesto Gulietta Fadda desde la década de 1990.

Queda así abierta la invitación para la discusión teórica, la investigación de campo y el desarrollo de herramientas y propuestas concretas que les permitan a las comunidades avanzar en la dirección presentada en este trabajo.

\section{Bibliografía}

Albornoz, B. (2007). “Experiencias de gobierno electrónico en la región andina: Una visión de conjunto", en Albornoz, B. y M. Rivero (comp.). Experiencias andinas de gobierno electrónico: La problemática de la participación ciudadana. Otawa, 
Canadá: Centro Internacional de Investigación para el Desarrollo (IDRC-CDRI), 291, 302, 303.

AndRADE, J. (enero-febrero-marzo del 2009). “Educación y tecnologías de información: Herramientas contra la pobreza en Venezuela". Educere. Año 13, núm. 44, 21-28.

ANDRAde, J. Y Y. Yedra (mayo-agosto del 2007). "Sistemas transparentes para gobiernos electrónicos eficientes". Enl@ce: Revista Venezolana de Información, Tecnología y Conocimiento. Año 4, núm. 2, 81-95.

Araya, R. (2005). “Internet, política y ciudadanía". Revista Nueva Sociedad 195. <http://www.nuso.org/upload/ articulos/3239_1.pdf>. [Consulta: 23 de enero del 2009].

-. (2004). "Tres perspectivas para observar el gobierno electrónico", en Araya, R. y M. Porrúa (comp.). América Latina Puntogob. Santiago de Chile: Flacso-Chile, 23-39.

Backus, M. (2001). "E-Governance and developing countries". Londres. <http://www.ftpiicd.org/files/research/reports/report3.pdf>. [Consulta: 11 de agosto del 2009].

Carballo, Y.; Cattafi, R.; Sanoja, A. y N. Zambrano (2006). "Gobierno electrónico en Venezuela". Universidad Central de Venezuela, Facultad de Ciencias, Escuela de Computación. <http://www.avw. org.ve/pdf/UCV.pdf>. [Consulta: 13 de agosto del 2009].
Cardon, D. (2005). “La innovación por el uso", en Ambrosi, A.; Peugeot, V, y D. Pimienta (comp.). Palabras en Juego: Enfoques multiculturales sobre las sociedades de la información. Guadalajara (México): C. \& F. Éditions.

Cendrós, J.; Durante, C. y J. Fermín (2004). "Factores estratégicos para desarrollar el gobierno electrónico en las alcaldías de Venezuela". Revista de Ciencias Humanas y Sociales. Vol. 20, núm. 45. Maracaibo.

Coleman, S. y J. Gotze (2001). Bowling together: Online public engagement in policy deliberation. Londres: Hansard Society.

Criado, I.; Ramilo, M. y M. Salvador (2002). “La necesidad de teoría(s) sobre gobierno electrónico. Una propuesta integradora". XVI Concurso de Ensayos y Monografías del CLAD sobre Reforma del Estado y Modernización de la Administración Pública "Gobierno Electrónico". Caracas. <http://www. cenit.gob.ve/cenitcms/servlet/ com.mvdcomm.cms.andocasociado?63,88>. [Consulta: 9 de agosto del 2009].

De Barry, H. y H. Palmar (2010). “Trámites en línea disponibles en la ventanilla única del gobierno electrónico de Venezuela". <https:// docs.google.com/fileview?id=0B6br Qc4sXIxhYjRiZjMwOGMtNWQxY i00MTFhLWIxZWEtYzE5YTExNG NmNTA2\&hl=en>. [Consulta: $17 \mathrm{de}$ agosto del 2010]. 
García, S. y M. Santiago (2009). “Tecnologías de comunicación e información y la participación democrática en Venezuela". <http:// www.scribd.com/doc/17549865/ TIC-y-Participacion-Democraticaen-Venezuela>. [Consulta: 24 de julio del 2010].

GartNer, G. (2000). Singapor's E-Government initiative. Stanford, Connecticut: Gartnet. First Take.

Gonzalo, M. (2006). “El gobierno electrónico venezolano: En busca de una democracia participativa y protagónica". Cibersociedad. $<$ http://www.cibersociedad.net/ congres2006/gts/comunicacio. php?id=1080>. [Consulta: 23 de enero del 2009].

—. (2005). “Uso y apropiación de los infocentros instalados en el Estado Zulia". Tesis para optar el título de magíster en ciencias de la comunicación. Universidad del Zulia, Venezuela.

Gotopo, G.; Leal, E. y J. Oliveros (2010). “Oferta de gobierno electrónico en sitios web del Poder Ejecutivo venezolano". <https:// docs.google.com/fileview?id=0B6br Qc4sXIxhYjRiZjMwOGMtNWQxY i00MTFhLWIxZWEtYzE5YTExNG NmNTA2\&hl=en>. [Consulta: 23 de agosto del 2010].

Holmes, D. (2001). @.gob. Estrategias para el uso eficiente de internet en el gobierno. Traducción: N. Brealey. México D.F.: McGraw-Hill Interamericana.
Kaufman, E. (2007). "Sobre políticas y modelos de gestión para el gobierno electrónico y la sociedad de la información y el conocimiento. Las recetas ajenas y los posibles desarrollos propios", en Kaufman, E. (comp.). Políticas públicas y tecnologías. Buenos Aires: La Crujía Ediciones.

Kaufman, E. y S. Piana (2007). "Algunas aclaraciones sobre gobierno electrónico y sociedad de la información y el conocimiento", en Kaufman, E. (comp.). Políticas públicas y tecnologías. Buenos Aires: La Crujía Ediciones.

Montilla, A. y A. PÁez (2010). “Experiencias de participación ciudadana a través de internet en centros de gestión parroquial de Venezuela". Quórum Académico. Vol. 7, núm. 1, 11-44.

—. (2007). "Participación ciudadana a través del Gobierno Electrónico. Caso: Centro de Gestión Parroquial 'Jesús Enrique Lossada'”. Enl@ce. Vol. 4, núm. 3, 19-40.

Morales, E. (2004). “¿Cuál comunicación para qué democracia?". Utopía y Praxis Latinoamericana. Año 9, núm. 24, 66-77.

-. (2002). "Democracia, política y comunicación. Enfoques latinoamericanos". Tesis para optar el título de doctor en ciencia política. Universidad del Zulia, Venezuela.

—. (1995). "Estado, sociedad civil y crisis de la democracia". Tesis 
para optar al título de magister scientiarum en ciencia política, mención política latinoamericana. Universidad del Zulia, Venezuela.

Naciones Unidas (2010). “Interactive Knowledge Base (UNKB) of global e-government information and data". <http://www2.unpan.org/ egovkb/>. [Consulta: 1 de septiembre del 2010].

Neüman, M. (junio del 2008). “La apropiación social como práctica de resistencia y negociación con la modernidad". Anuario ININCO. Vol. 20, núm. 1. Caracas, pp. 47-78.

-. (5-8 de octubre del 2003). "Cuando estar 'enchufados' adquiere sentido: Reflexiones desde la experiencia venezolana". XI Encuentro Latinoamericano. Puerto Rico.

ОсноА, Н. (2006). “Gobierno electrónico y contraloría social en las gobernaciones venezolanas". Enl@ce. Año 3, núm. 2, 11-28.

Окот-UMA, R. (2001). “Electronic governance: re-inventing good governance". Londres. <http://haspp. axesnet.com/contenido/documentos/Okot-Uma.pdf>. [Consulta: 9 de agosto del 2009].

Páez, Á. (2011). Gobierno electrónico de abajo hacia arriba: Una propuesta de Venezuela. Santa Fe: Institute for Analytic Journalism. Vol. 1.

- (2010). "Hacia una teoría venezolana sobre gobierno electrónico". El gobierno electrónico en Venezuela. Balance y perspectivas. Mérida: Universidad de Los Andes.
PÁez, Á. y H. Castañeda (2007). “Evolución del gobierno electrónico local en Venezuela". Temas de Comunicación 15. Caracas: Universidad Católica Andrés Bello, 173-190.

PÁez, A.; Iribarren, I. y M. Neüman (octubre-noviembre del 2003). "Gobierno electrónico y administración pública local". Razón y Palabra. Año 8, núm. 35. <http:// www.razonypalabra.org.mx/anteriores/n35/apaezmneuman.html .

Petrizzo, P. (2005). “Participación ciudadana y tecnologías de la información y la comunicación: Hacia una administración pública relacional". Nueva Sociedad 195. <http://www. nuso.org/upload/articulos/3241_1. pdf $>$. [Consulta: 23 de enero del 2009].

Reilly, K. (2004). "Estrategias de E-Gobierno en ocho países de América Latina", en Araya, R. y M. Porrúa (comp.). América Latina Puntogob. Santiago de Chile: FlacsoChile.

Reilly, K. y R. Echeverría (2003). “El papel del ciudadano y de las OSC en el e- Gobierno. Un estudio de gobierno electrónico en ocho países de América Latina y el Caribe". Monitor de políticas TIC y derechos en internet en América Latina y el Caribe. <http://lac.derechos.apc. org/investigacion/e-gobierno.pdf>. [Consulta: 22 de junio del 2010].

Rincón, D. y M. Romero (2003). “Las tecnologías de información y comunicación en el gobierno electró- 
nico de la alcaldía de Maracaibo". Revista de Ciencias Humanas y Sociales. Vol. 19, núm. 42.

Salazar, R. (2008). Quién es el sujeto insumiso. México: Enciclopedia Internacional de Ciencias Sociales.
(2005). “Ciudadanías emancipadas y emancipatorias", en Salazar, Robinson y Paula Lengüita (comp.). Democracia emancipatoria. México: Libros en Red. 